\title{
Correction
}

\section{Correction: Sepers et al., "Endocannabinoid-Specific Impairment in Synaptic Plasticity in Striatum of Huntington's Disease Mouse Model"}

In the article, "Endocannabinoid-Specific Impairment in Synaptic Plasticity in Striatum of Huntington's Disease Mouse Model" by Marja D. Sepers, Amy Smith-Dijak, Jeff LeDue, Karolina Kolodziejczyk, Ken Mackie, and Lynn A Raymond, which appeared on pages $544-554$ of the January 17, 2018 issue, the authors have become aware of an error in Figure 7B, where the legend indicating treatment in YAC128 iSPN was inadvertently reversed. The revised figure correctly shows untreated YAC128 iSPN with blue circles and YAC128 iSPN treated with URB597 with gray circles. In addition, we have changed the legend to clarify that the same group of untreated YAC128 iSPN is presented in all panels for comparison with drug treatments. The authors apologize for the error. The updated figure and figure legend can be found below.

A

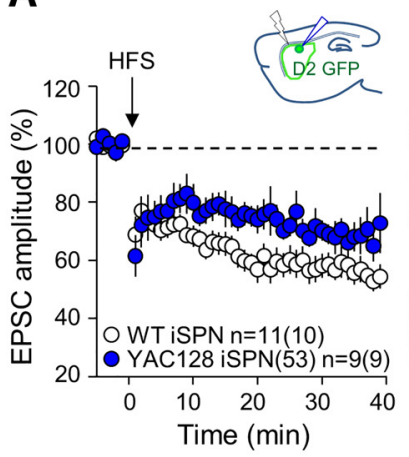

D

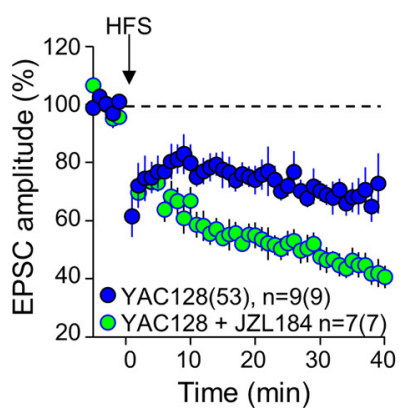

B

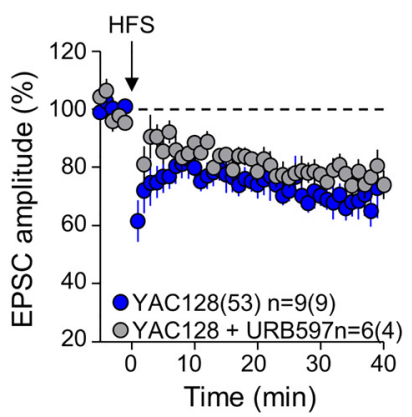

C

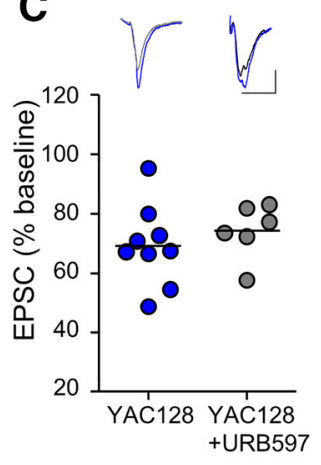

E

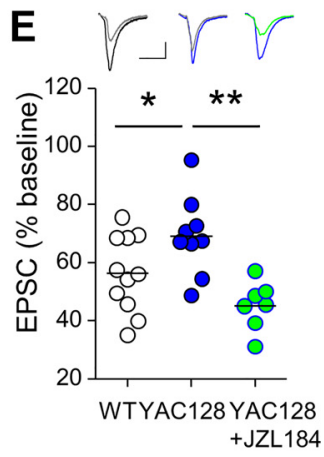

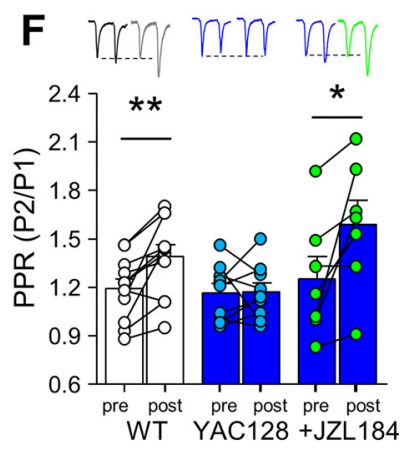

Figure 7. HFS-induced long-term depression rescued in YAC128 by increasing 2-AG levels. A, Time course summary of normalized EPSC amplitude after HFS at time 0 in 1 month-old WT and YAC128(53) in indirect pathway SPNs (iSPNs). The same group of untreated YAC128(53) iSPN are shown in subsequent panels for comparison with drug treatment. $\boldsymbol{B}$, Time course summary of untreated YAC128(53) iSPNs and YAC128(53) iSPNs treated with the FAAH inhibitor URB597 showing no difference in response to HFS. C, Summary and representative traces showing no difference in YAC128 iSPNs treated with URB597 $(1 \mu \mathrm{M})$ compared with untreated YAC128 iSPNs. D, Time course summary of untreated YAC128 iSPNs and YAC128 iSPNs treated with the MAGL inhibitor JZL184 $(10 \mu \mathrm{M}$ ) showing normalized EPSC amplitude after HFS. $\boldsymbol{E}$, Mean responses (showing data at $35 \mathrm{~min}$ after LTD induction relative to baseline in each experiment from the time course summary in $\boldsymbol{A}$ for WT and untreated YAC128, and from D for YAC128 treated with JZL184) illustrate significantly greater reduction in WT compared with YAC128 iSPNs and in YAC128 iSPNs treated with JZL184 compared with untreated YAC128 iSPNs. Representative traces shown in inset: pre- and post-HFS in WT, YAC128, and YAC128 treated with JZL184. Scale: 100 pA, 20 ms. F, Paired-pulse ratio (PPR) of EPSC after HFS is increased in WT iSPNs and YAC128 treated with JZL184, but not untreated YAC 128 iSPNs.

DOI: 10.1523/JNEUROSCI.1804-18.2018 